\title{
Abnormal Collateral Vessels in Behçet's Disease Revealed by Gastrointestinal Bleeding Scintigraphy with ${ }^{99 m}$ Tc-RBC
}

\author{
Jingnan Wang1,2, Yaping Luo ${ }^{1,2}$, Xiaona Jin1,2, Chunyuan Zhao1,2 and \\ Fang $\mathrm{Li}^{1,2 *}$ \\ ${ }^{1}$ Department of Nuclear Medicine, Chinese Academy of Medical Sciences and Peking \\ Union Medical College Hospital, P.R. China \\ ${ }^{2}$ Beijing Key Laboratory of Molecular Targeted Diagnosis and Therapy in Nuclear \\ Medicine, P.R. China
}

*Corresponding author: Fang Li, Department of Nuclear Medicine, Chinese Academy of Medical Sciences and Peking Union Medical College Hospital, P.R. China, Tel: 86-10-69155502; Email: lifang@pumch.com

\begin{abstract}
A 36-year old male who had a history of Behçet's disease for 9 years was found with occlusion and stenos is of multiple vessels including left subclavicular artery, aortic arch, bilateral brachiocephalic veins, superior vena cava, inferior vena cava, bilateral renal veins, iliac veins and deep veins in bilateral lower extremities. CTA showed collateral vascular circulation in mediastinum, bilateral axilla, chest wall and abdominal wall, and in the upper abdomen around the liver and in retroperitoneal region due to vascular stenos is and obstruction. 5 days ago, he presented with hematochezia due

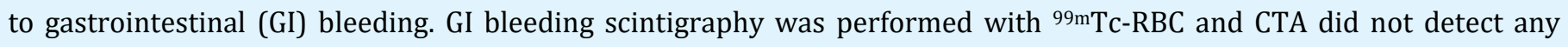
active GI bleeding during the study; instead, the GI bleeding scintigraphy found. Abnormal vascular pathway and tortuous linear vessel maess in the left upper quadrant of the abdomen, that was consistent with the dilated collateral vessels detected in CTA caused by Behçet's disease.
\end{abstract}

Keywords: Gastrointestinal Bleeding Scintigraphy; Behçet’s Disease; Collateral Vessels

\section{Case Report}

A 36-year-old man presented with recurrent oral and genital ulcerations for 9 years. He had repeated episodes of deep venous thrombosis in lower extremities in recent 5 years, and anticoagulation therapy with war far in was intermittently used. Two years ago, his symptom with genital ulcerations was exacerbated; mean while, erythema nodosum, redness and edema occurred in his lower extremities. Ultrasound showed deep venous thrombosis in bilateral lower extremities, together with thrombosis in both right and left subclavicular veins. He was then diagnosed as Behçet's disease after hospitalization for further evaluation of the disease. In contrast-enhanced CT, there were multi plefilling defects in the left subclavicular artery, aortic arch, bilateral brachiocephalic veins, superior vena cava (SVC), inferior vena cava (IVC), bilateral renal veins and iliac veins 
(Figure 1A-B). CTA with three-dimensional volume rendering image demons trated mal formed vessels in mediastinum, bilateral axilla, left chest wall and abdominal wall, and in the upper abdomen around the liver and in retroperitoneal region (Figure 1C-D). These abnormal vessels were considered to be collateral circulation due to vascular stenos is and obstruction caused by thrombosis from Behçet's disease. Thereafter, the patient was treated with prednisone, cyclophosphamide, thalidomide, and leflunomide against Behçet's disease. Anticoagulation treatment with war far in with regular monitoring of INR was also started.

5 days ago, the patient presented with hematochezia (1-2 times per day, $50-100 \mathrm{~mL}$ per time) and abdominal pain. Laboratory tests showed haemoglobin level dropped from $120 \mathrm{~g} / \mathrm{L}$ to $99 \mathrm{~g} / \mathrm{L}$. Physical examination and gastros copy were unremarkable, and abdominal CTA did not detect the bleeding site. Then the patient was referred for gastrointestinal (GI) bleeding scintigraphy in order to localize the site of GI bleeding. GI bleeding scintigraphy was performed with 99mTc-RBC (in vitro labeling method).After administration of $99 \mathrm{~m} \mathrm{Tc}-\mathrm{RBC}$ (20mCi), dynamic planar images of the abdominal and pelvis were acquired to 30 minutes with a dual-head gamma camera( $2 \mathrm{~s} /$ frame $\times 30$ frames, $1 \mathrm{~min} /$ frame $\times 29$ frames). Late planar images were acquired at $1 \mathrm{~h}, 3 \mathrm{~h}, 5 \mathrm{~h}$ and $22 \mathrm{~h}$ time points. In the early angiographic images (Figure $2 \mathrm{~A}$ ), abnormal vascular pathway was demonstrated: after intravenous injection through the left median cubital vein, the radiotracer directly went to abdominal aorta via the collateral vessels in the left chest wall, instead of going to pulmonary circulation first. In the following dynamic and late images (Figure 2B), there was tortuous linear uptake in the left upper quadrant of the abdomen. The location and shape of the abnormal uptake did not change but the intensity decreased in the late phase. These findings were consistent with the dilated collateral vessels detected in CTA. No active GI bleeding was detected until $22 \mathrm{~h}$ after injection.

After discontinuation of war far in and conservative treatment with fasting, acid suppression and rehydration for GI bleeding, his symptoms of hematochezia relieved. The Hgb level remained stable at 106-109 $\mathrm{g} / \mathrm{L}$, and feces occult blood test was negative. The GI bleeding was considered under control, and then the patient was discharged.

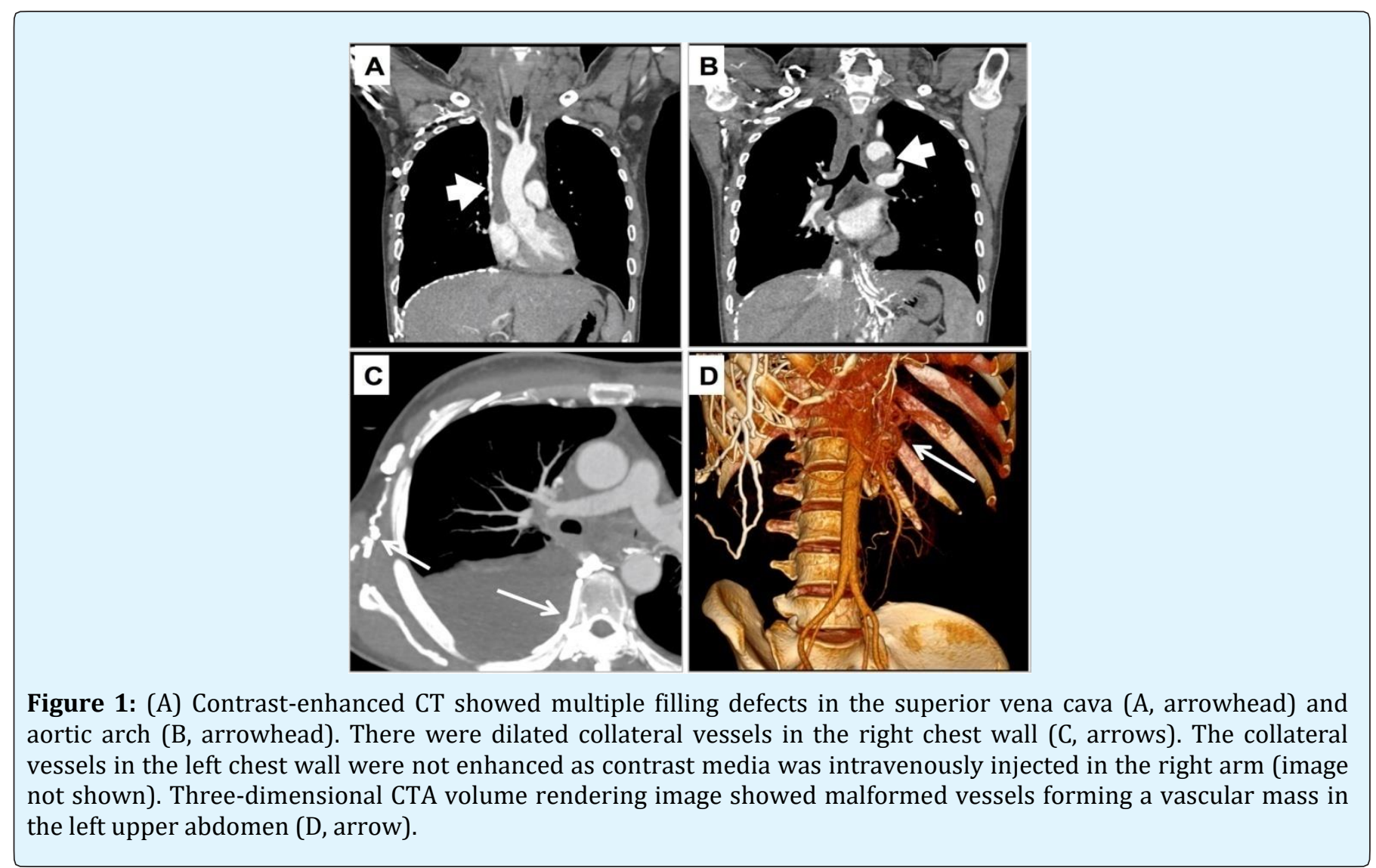

Fang Li, et al. Abnormal Collateral Vessels in Behçet's Disease Revealed by Gastrointestinal Bleeding Scintigraphy with $99 \mathrm{mTc}$-RBC. Int J Nuclear Med Radioactive Subs 2018, 1(1): 000104. 

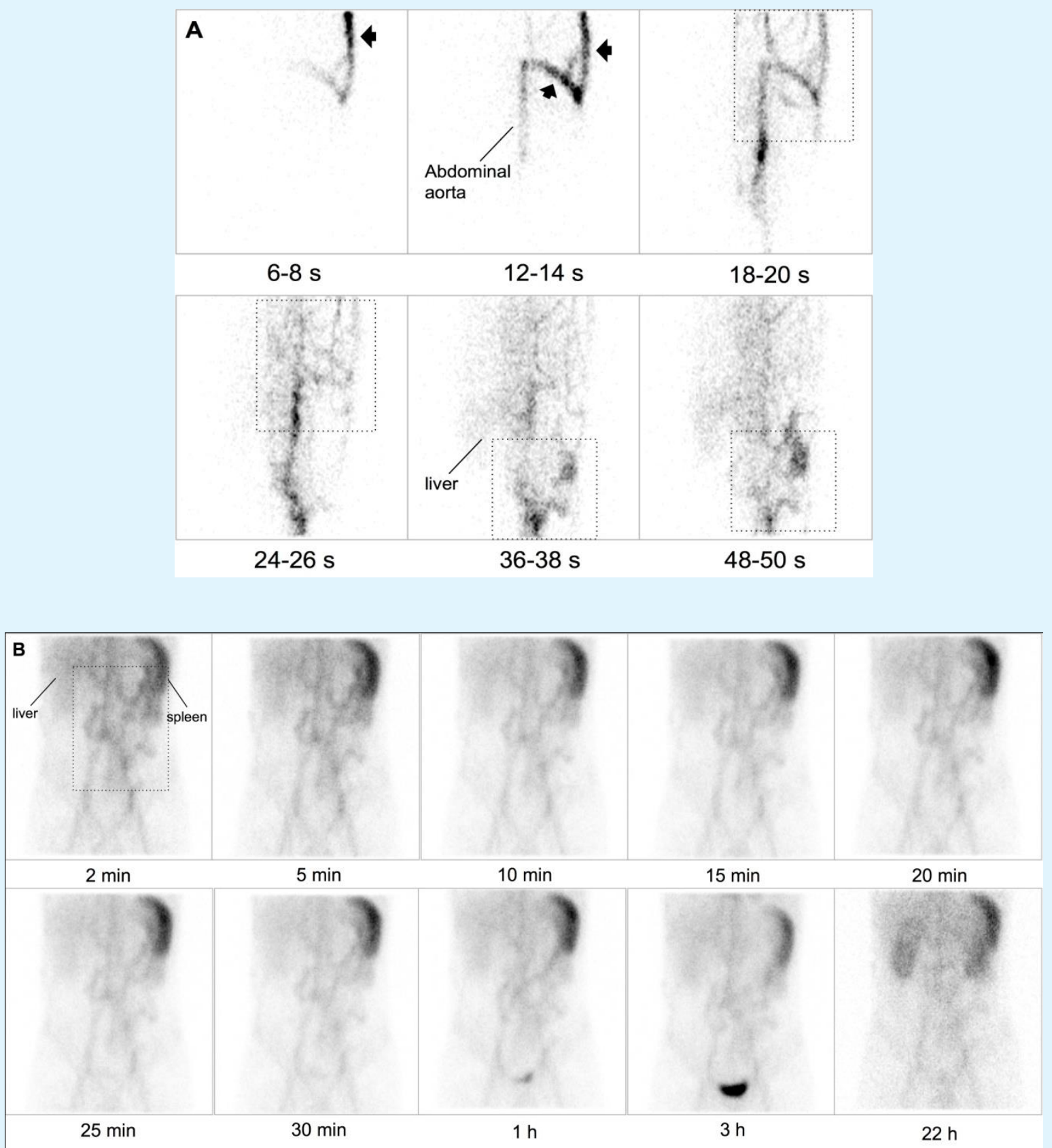

Figure 2: Gastrointestinal (GI) bleeding scintigraphy of the 36-year-old male patient with Behçet's disease. (A)Early angiographic images $(2 \mathrm{~s} /$ frame $\times 30$ frames) showed abnormal visualization of collateral vessels in the left chest wall (arrowhead) directly after intravenous injection through the left median cubital vein. Then abdominal aorta and liver was sequentially visualized. There were malformed and dilated vessels in both left chest and left upper quadrant of the abdomen (dotted block).

(B) Dynamic and late planar images showed tortuous linear uptake in the left upper quadrant of the abdomen (dotted block), the location and shape of which remained unchanged until $22 \mathrm{~h}$ after injection. No active GI bleeding was detected. Notice the change of field of view in angiographic phase and the subsequent dynamic and late images. 


\section{International Journal of Nuclear Medicine \& Radioactive Substances}

\section{Discussion}

Behçet's disease is a chronic multisystem disorder characterized by recurrent oral and genital ulcerations, uveitis and erythema nodosum. The etiology and pathogenesis remain obscure, vasculitis is the main pathologic lesion with a tendency to venous thrombus formation in Behçet's disease. Additionally, circulating auto anti bodies to human oral mucous membranes are found in half of the patients, which is responsible for recurrent oral ulcerations. Vascular diseases manifestations in Behçet's disease include venous thrombotic occlusions, arterial occlusions, pseudoaneurysm, and so on [1]. The common type of venous involvement deep venous thrombosis, usually found in (IVC), (SVC) and cerebral veins [1,2], which could cause obstruction of the affected vessels. In our case, the patient presented with repeated episodes of venous thrombosis in bilateral lower extremities, left sub clavicular artery, aortic arch, bilateral brachiocephalic veins, (SVC), (IVC), bilateral renal veins, iliac veins, and deep veins in bilateral lower extremities. Left subclavicular artery and aortic arch were also affected. Due to occlusion and stenos is of these vessels, malformed collateral vessels were formed in media stinum, bilateral axilla, left chest wall and abdominal wall, and in the upper abdomen around the liver and in retroperitoneal region to ensure the blood supply in the body.

Presentations of Behçet's disease are common, including abdominal pain, nausea, vomiting, diarrhea and GI bleeding. GI bleeding in Behçet's disease may be caused by the mucosal inflammation, ulcer formation, rupture of mesenteric aneurysm, variceal bleeding, and anticoagulant therapy [3].

GI bleeding can be evaluated by scintigraphy. This examination could determine whether the bleeding is active, localize the bleeding bowel segment and estimate

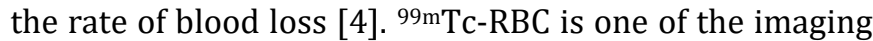
agent for GI bleeding that was first described by Win Zelberg, et al. [5]. The long-time stability of ${ }^{99 \mathrm{~m}} \mathrm{Tc}-\mathrm{RBC}$ in the blood pool allows for long scanning duration, increasing the likelihood for detecting intermittent bleeding. In previous studies, a minimum bleeding rate of $0.04 \mathrm{~mL} / \mathrm{min}$ (at least $2-3 \mathrm{~mL}$ amount of bleeding) could be detected [6], and the sensitivity of detecting GI bleeding has been reported to be $93 \%$ with $99 \mathrm{mTc}-\mathrm{RBC}$ [7]. However, in clinical practice, prior treatment toward GI bleeding may hamper the detection rate of GI bleeding scintigraphy. The highest detection yield is produced when this study is performed as soon as possible after patients arriving in the emergency room or on admission.
Other false-negative results occur when the bleeding is not active at the time of the study, or the amount and rate of the bleeding are lower than the detection efficiency. In addition, infrequent image acquisition or insufficient scanning duration could be other reasons.

Misinterpretation of GI bleeding scintigraphy with

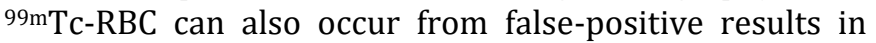
ectopic spleen, neoplasm, angiodysplasia, abscess, colitis, Meckel's diverticulum, and reproduction organs [8]. In our case, the patient presented hematochezia and abdominal pain caused by GI bleeding. However, neither CTA nor GI bleeding scintigraphy with ${ }^{99 \mathrm{~m} T c-R B C}$ detected the bleeding site. The possible reason was prior.

Treatments including discontinuation of war far in, water fasting, acid suppression and rehydration once the patient arrived the emergency room, so that there might not be active GI bleeding when the imaging study was performed. His symptoms then relieved, the shape and color of stool was normal and feces occult blood test became negative. The, Hgb level was also stable at 106$109 \mathrm{~g} / \mathrm{L}$, suggesting recovery of GI bleeding. The exact cause and site of GI bleeding and negative feces occult blood. Remained unknown until the patient was discharged.

Although active GI bleeding was not detected in this

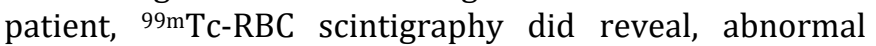
vascular pathways and tortuous vessel mass in the abdomen, consistent with the dilated collateral vessels detected in CTA. In the presence of IVC or SVC obstruction, blood from the extremities and abdominal organs should be diverted into various main and accessory collateral svessels. Dilated veins including azygos or hemiazygos vein, vertebral venous plexus, internal and external mammary vein, superficial thoracicab dominal vein, and superficial veins of viscera would be served as collateral pathways when there are chronic obstructions [9-11]. CTA and digital subtraction angiography could identify the exact anatomical localizations of the obstruction and collateral vascular pathways the current case, the GI bleeding scintigraphy with ${ }^{99} \mathrm{mTc}$-RBC may evaluate if the GI bleeding was active or not. Apart from may provides additional information to formation of collateral vessel in Behçet's disease as ${ }^{99 \mathrm{~m} T c-}$ RBC being a blood pool imaging agent.

\section{References}

1. Tascilar K, Melikoglu M, Ugurlu S, Sut N, Caglar E, et al. (2014) Vascular involvement in Behcet's syndrome: a retrospective analysis of associations 


\section{International Journal of Nuclear Medicine \& Radioactive Substances}

and the time course. Rheumatology (Oxford) 53(11): 2018-2022.

2. Fei Y, Li X, Lin S, Song X, Wu Q, et al. (2013) Major vascular involvement in Behcet's disease: a retrospective study of 796 patients. Clin Rheumatol 32(6): 845-852.

3. Skef W, Hamilton MJ, Arayssi T (2015) Gastrointestinal Behcet's disease: a review. World J Gastroenterol 21(13): 3801-3812.

4. Dam HQ Brandon DC, Grantham VV, Hilson AJ, Howarth DM, et al. (2014) The SNMMI procedure standard/EANM practice guideline for gastrointestinal bleeding scintigraphy 2.0. Journal of nuclear medicine technology 42(4): 308-317.

5. Winzelberg GG, McKusick KA, Strauss HW, Waltman AC, Greenfield AJ (1979) Evaluation of gastrointestinal bleeding by red blood cells labeled in vivo with technetium-99m. J Nucl Med 20(10): 10801086.

6. Thorne DA, Datz FL, Remley K, Christian PE (1987) Bleeding rates necessary for detecting acute gastrointestinal bleeding with technetium-99m- labeled red blood cells in an experimental model. J Nucl Med 28(4): 514-520.

7. Bunker SR, Lull RJ, Tanasescu DE, Redwine MD, Rigby J, et al. (1984) Scintigraphy of gastrointestinal hemorrhage: superiority of $99 \mathrm{mTc}$ red blood cells over $99 \mathrm{mTc}$ sulfur colloid. AJR Am J Roentgenol 143(3): 543-548.

8. Grady E (2016) Gastrointestinal Bleeding Scintigraphy in the Early 21st Century. J Nucl Med 57(2): 252-259.

9. Temizoz O, Genchellac H, Yekeler E, Demir MK, Unlu E, et al. (2010) CT-angiographic demonstration of hepatic collateral pathways due to superior vena cava obstruction in Behcet disease. Diagn Interv Radiol 16(4): 302-305.

10. Stanford W, Jolles H, Ell S, Chiu LC (1987) Superior vena cava obstruction: a venographic classification. AJR Am J Roentgenol 148(2): 259-262.

11. Kapur S, Paik E, Rezaei A, Vu DN (2010) where there is blood, there is a way: unusual collateral vessels in superior and inferior vena cava obstruction. Radiographics 30(1): 67-78. 